\title{
Análisis de la Viabilidad Ambiental de la Utilización de Morteros Fabricados con Polvo de Vidrio en la Estabilización de Suelos
}

\author{
María I. Mas(1), Eva M. García(2), Luis J. Marco(1) y Jaime de Marco(3) \\ (1) Universidad Politécnica de Madrid, Escuela Técnica Superior de Ingeniería Civil, Departamento de \\ Ingeniería Civil: Construcción, Infraestructura y Transporte, Alfonso XII 3-5, 28014 Madrid - España. \\ (e-mail:mariaisabel.mas@upm.es; luisjaime.marco@upm.es) \\ (2) Universidad Politécnica de Madrid, Escuela Técnica Superior de Ingeniería Civil, Departamento de \\ Ingeniería Civil: Hidráulica y Ordenación del territorio, Alfonso XII 3-5, 28014 Madrid - España. \\ (e-mail: evamaria.garcia@upm.es) \\ (3) Beroa Bierrum, 6 Water End Barns, Water End, Eversholt MILTON KEYNES, Bucks MK 17 9EA- United \\ Kingdom (e-mail: jaime.demarco@beroa-altac.com)
}

Recibido May. 4, 2016; Aceptado Jun. 16, 2016; Versión final Jul. 28, 2016, Publicado Oct. 2016

\begin{abstract}
Resumen
En este trabajo se realiza un análisis del impacto ambiental producido por la utilización de morteros cuyo ligante son desechos de polvo de vidrio. Dentro del concepto de sostenibilidad, la ingeniería civil debe desarrollar su capacidad para utilizar materiales de construcción que reutilicen productos de desecho, cuyo destino final son vertederos controlados y por otra parte que minimicen el impacto negativo en el medio ambiente a la hora de su puesta en obra. Para este fin, se han realizado ensayos de filtración sobre probetas de morteros fabricados con residuos de polvo de vidrio como ligante para identificar la migración o emisión de elementos lixiviados. La realización de estos ensayos ha dado como resultado que el único elemento liberado en forma apreciable es el sodio (procedente fundamentalmente del vidrio). Todos los demás elementos son liberados en cantidades inferiores a un $\mathrm{mg} / \mathrm{l}$ por lo que no representan peligro ambiental.
\end{abstract}

Palabras clave: Lixiviados; mortero; polvo de vidrio; Ligante.

\section{Environmental Viability Assessment of Using Mortar Containing Glass Powder in Soil Stabilization}

\begin{abstract}
This paper presents the analysis of the environmental impact caused by the use of mortar whose binder is made with waste glass powder. Within the concept of sustainability, civil engineering should develop their ability to use building materials reusing waste products, whose final destination are controlled landfills and secondly to minimize the negative impact on the environment when they are put into work. To this end, tests have been conducted on specimen's filtration mortars containing waste glass powder as binder migration to identify potentially dangerous emission of harmful elements. The realization of these trials has resulted in that sodium is the only element liberated in detectable amounts (principally from the glass). All other elements are released in quantities lower than one $\mathrm{mg} / \mathrm{l}$, which represent no hazard to the environment.
\end{abstract}

Keywords: leached; mortar; glass power; binder 


\section{INTRODUCCIÓN}

El rápido crecimiento y desarrollo de los países industrializados, la creencia de que los recursos disponibles son ilimitados y el excesivo crecimiento demográfico, unido a que los impactos que producimos sobre el entorno son, generalmente, tan a largo plazo que no se aprecian directamente, hacen que los efectos de nuestro modelo de desarrollo lo sufran las generaciones venideras. Lo cierto es que los recursos son limitados, la naturaleza tiene unos topes de producción de materias y servicios así como de absorción de residuos (Rodríguez, et al, 2010). La ingeniería civil desde los tiempos más antiguos ha impulsado el desarrollo de nuestra sociedad a través de la construcción de diferentes tipos de infraestructuras. Pero este desarrollo ha provocado severos daños ambientales debido a la gran cantidad de recursos naturales demandados, así como a la contaminación producida. Ante estas perspectivas, los temas relacionados con la preservación del medioambiente empiezan a movilizar importantes sectores sociales, tanto del ámbito gubernamental como de la propia sociedad (Burgueño 2007).

En el momento actual se debe trabajar para un Desarrollo Sostenible y la ingeniería civil debe aportar dentro del concepto de sostenibilidad toda su capacidad (Valdés et al, 2007). Dentro de esta línea de trabajo se encuentra el presente artículo. Por una parte, se contribuye a reducir los residuos últimos procedentes del proceso del reciclado de vidrio de todo tipo de envases y embalajes de la industria vidriera y cerámica que serían depositados en vertederos controlados (Bashart y Ghassan, 2008). Por otra, se disminuye la cantidad de gases de efecto invernadero emitidos puesto que parte del cemento utilizado en la elaboración de los morteros se sustituye por polvo de vidrio, con el consiguiente beneficio medioambiental y económico (Ahmad y Aimin, 2003).

Estudios anteriormente realizados (Marco et al, 2012) constataron que el uso de morteros fabricados con polvo de vidrio es viable desde el punto de vista de la resistencia a compresión en la estabilzación de suelos. Como complemento a estos, el presente estudio se dirige a evaluar la viabilidad ambiental de dichos morteros. Con este propósito, se realizan ensayos de permeabilidad para posteriormente realizar un estudio de los lixiviados recogidos, habida cuenta de que en la composición del polvo de vidrio empleado, puede haber algunos elementos que son potencialmente contaminantes para los suelos o acuíferos, los llamados elementos traza (Martinez, et al, 2013). Entre los que destacan: Mo, Pd, Ag, As, Cd, Co, Cu, Pb, Sn y TI. Por lo tanto, resulta importante estudiar el nivel de toxicidad que se genera cuando se utilizan morteros fabricados con polvo de vidrio en la estabilización de suelos, dado que el material va a ser aplicado en el exterior, dónde el agua de lluvia, el agua superficial o las aguas subterráneas, pueden ser responsables de que ocurran procesos de lixiviación. Los resultados obtenidos se han comparado con la legislación pertinente, determinando que la inclusión del polvo de vidrio en la fabricación de morteros hace viable su uso en la estabilización de suelos.

\section{MATERIALES Y METODOS}

Para la realización de los ensayos se han caracterizado tanto el cemento como el polvo de vidrio usado como ligante. Este último proviene de la molienda de los residuos de la industria cerámica (fritas) y de los residuos últimos del reciclaje de envases y embalajes de vidrio, que por sus características no pueden ser ya utilizados en la industria vidriera y son depositados en vertederos de inertes.

El cemento utilizado fue un cemento Pórtland comercial (CEM I 52,5 R), proveniente de la fábrica de Cementos Pórtland en Chinchón (Madrid). La letra R indica una resistencia a corto plazo elevada. Tiene una masa volumétrica de $3.12 \mathrm{~g} / \mathrm{cm}^{3}$, una superficie específica de $4.440 \mathrm{~cm}^{2} / \mathrm{g}$ y un color gris verdoso. Sus características granulométricas aparecen en la tabla 1 y en la tabla 2 la composición química

Tabla 1: Granulometría del cemento CEM I 52,5 R (Elaboración propia)

\begin{tabular}{|c|c|}
\hline Diámetro de las partículas en $(\mu \mathrm{m})$ & Fracción volumétrica en $(\%)$ \\
\hline$<8$ & 41.5 \\
\hline$<96$ & 99.7 \\
\hline
\end{tabular}

El polvo de vidrio utilizado proviene de la molienda de los residuos de la industria cerámica (fritas) y de envases y embalajes procedentes de la recogida selectiva de basuras que por sus características (elevado porcentaje de papel, corcho o plástico adheridos...) no pueden reutilizarse. Han sido molidos en un molino de barras equipado con 15 barras de 3 diámetros diferentes y con diferentes tiempos de molienda.

La granulometría del polvo de vidrio ha sido determinada por tres diámetros característicos de $d_{10}$, $d_{50}$, d $d_{90}$, que constituyen la dimensión de las partículas de la muestra para la cual el $10 \%$, el $50 \%$ y el $90 \%$ de las mismas, poseen un diámetro inferior a ese valor. En la tabla 3 aparecen las características granulométricas del polvo de vidrio en función del tiempo de molienda. 
Tabla 2: Composición química del CEM I 52,5 R (Elaboración propia)

\begin{tabular}{|l|c|c|}
\hline \multicolumn{1}{|c|}{ Componente } & Óxidos Componentes & Porcentaje (\%) \\
\hline Cal combinada & $\mathrm{CaO}$ & 65 \\
\hline Sílice & $\mathrm{SiO}_{2}$ & 19 \\
\hline Alúmina & $\mathrm{Al}_{2} \mathrm{O}_{3}$ & 5.5 \\
\hline Hierro & $\mathrm{Fe}_{2} \mathrm{O}_{3}$ & 2.65 \\
\hline Cal libre & $\mathrm{CaO}$ & 0 \\
\hline Azufre & $\mathrm{SO}_{3}$ & 2 \\
\hline Magnesio & $\mathrm{MgO}_{2}$ & 2 \\
\hline \multirow{2}{*}{ Álcali } & $\mathrm{Na}_{2} \mathrm{O}$ & 0.15 \\
\hline Pérdida al fuego & $\mathrm{K}_{2} \mathrm{O}$ & 0.7 \\
\hline Residuo insoluble & P.F. & 2 \\
\hline
\end{tabular}

Tabla 3: Caracterización granulométrica del polvo de vidrio en función de la duración de la molienda (Elaboración propia)

\begin{tabular}{|l|c|l|c|c|}
\hline Polvo de vidrio utilizado & Tiempo de molienda & $\mathrm{d}_{10}$ & $\mathrm{~d}_{50}$ & $\mathrm{~d}_{90}$ \\
\hline Polvo de vidrio 1 & $2 \mathrm{~h} \mathrm{30} \mathrm{m}$ & $2.92 \pm 0.01 \mu \mathrm{m}$ & $33 \pm 1 \mu \mathrm{m}$ & $110 \pm 3 \mu \mathrm{m}$ \\
\hline Polvo de vidrio 2 & $4 \mathrm{~h} 15 \mathrm{~m}$ & $1.96 \pm 0.01 \mu \mathrm{m}$ & $16 \pm 1 \mu \mathrm{m}$ & $59 \pm 2 \mu \mathrm{m}$ \\
\hline Polvo de vidrio 3 & $5 \mathrm{~h}$ & $1.65 \pm 0.01 \mu \mathrm{m}$ & $11 \pm 1 \mu \mathrm{m}$ & $43 \pm 2 \mu \mathrm{m}$ \\
\hline
\end{tabular}

En la realización del ensayo se han empleado lotes del valor $d_{50}$, ya que según Marco et al, (2012), la relación del tiempo de molienda con el diámetro de las partículas obtenido representa un óptimo desde el punto de vista la sostenibilidad. La composición química de los residuos de polvo de vidrio empleados en la molienda es la que se refleja en la tabla 4 (Marco, et al, 2012).

Tabla 4: Composición química del polvo de vidrio empleado en la elaboración del producto (Elaboración Propia)

\begin{tabular}{|c|c|c|}
\hline Oxido & Vidrio & Fritas \\
\hline $\mathrm{SiO}_{2}$ & 71.00 & 62.13 \\
\hline $\mathrm{Na}_{2} \mathrm{O}$ & 11.80 & 3.57 \\
\hline $\mathrm{K}_{2} \mathrm{O}$ & 0.60 & 3.49 \\
\hline $\mathrm{CaO}$ & 11.28 & 9.27 \\
\hline $\mathrm{MgO}$ & 1.40 & 0.40 \\
\hline $\mathrm{MnO}$ & 0.10 & 0.00 \\
\hline $\mathrm{Al}_{2} \mathrm{O}_{3}$ & 2.20 & 8.74 \\
\hline $\mathrm{Fe}_{2} \mathrm{O}_{3}$ & 1.60 & 0.24 \\
\hline $\mathrm{TiO}_{2}$ & 0.07 & 0.00 \\
\hline $\mathrm{P}_{2} \mathrm{O}_{5}$ & 0.05 & 0.15 \\
\hline $\mathrm{SO}_{3}$ & 0.00 & 0.00 \\
\hline $\mathrm{Cr}_{2} \mathrm{O}_{3}$ & 0.00 & 0.00 \\
\hline $\mathrm{Co}_{3} \mathrm{O}_{4}$ & 0.00 & 0.00 \\
\hline $\mathrm{ZnO}$ & 0.00 & 5.23 \\
\hline SrO & 0.00 & 0.10 \\
\hline $\mathrm{ZrO}_{2}$ & 0,00 & 0,07 \\
\hline $\mathrm{BaO}$ & 0.00 & 0.00 \\
\hline $\mathrm{PbO}$ & 0.00 & 0.00 \\
\hline $\mathrm{HfO}_{2}$ & 0.00 & 0.10 \\
\hline $\mathrm{Rb}_{2} \mathrm{O}$ & 0.00 & 0.10 \\
\hline $\mathrm{CdO}$ & 0.00 & 0.10 \\
\hline $\mathrm{B}_{2} \mathrm{O}_{3}$ & 0.00 & 6.31 \\
\hline PF / Volátil & 0.90 & 0.00 \\
\hline Total & 100 & 100 \\
\hline
\end{tabular}


Se ha preparado un mortero, según norma UNE-EN 196-1, con arena y ligante en una relación 2,75:1 y una relación agua/ligante de 0,52. Cabe reseñar que en la composición del ligante, el $80 \%$ es polvo de vidrio y el $20 \%$ es cemento Portland, con las características descritas en párrafos anteriores. Esta proporción de ligante tiene su base en los resultados previos obtenidos por los autores (Marco et al, 2012), para ensayos de resistencia a compresión realizados, dónde se constató que los mejores resultados en cuanto a resistencia a compresión para este tipo de morteros se obtenían, precisamente, para una sustitución de cemento por polvo de vidrio del $80 \%$.

Tras la elaboración del mortero, se hacen ensayos de permeabilidad de tres probetas por amasada y unidad de ensayo, adaptando la norma UNE-EN 12390-8 (permeabilidad bajo presión para hormigón endurecido) con el objetivo de estudiar la evolución de la permeabilidad del testigo a lo largo del tiempo así como evaluar los elementos presentes en los lixiviados procedentes de las aguas de filtrado recogidas. El número total de amasadas realizadas es 5 , considerándose por la experiencia de los autores suficiente a la hora de realizar este ensayo. Las mediciones han sido efectuadas sobre este mortero, conservado en medio endógeno a $20^{\circ} \mathrm{C}$ durante 100 horas. El testigo del mortero es un cilindro de $4.07 \pm 0.01 \mathrm{~cm}$ de longitud y $3.92 \pm 0.01 \mathrm{~cm}$ de diámetro. La presión de inyección del agua en el mortero se mantiene constante a 10 bares durante todo el ensayo.

Con el fin de evaluar los elementos lixiviados, se realiza una filtración al vacío. Las filtraciones se recogieron en fracciones sucesivas de $\pm 20 \mathrm{~cm}^{3}$, mediante filtro de vidrio de $0,7 \mu \mathrm{m}$ siendo examinadas a través de la espectrometría de absorción atómica y por espectrometría de masa (ICP-MS).

\section{RESULTADOS Y DISCUSION}

Los resultados se exponen en dos subsecciones: en una se estudia el caudal filtrado y la permeabilidad de la muestra y en la otra se examinan los elementos arrastrados en la misma.

Caudal filtrado y permeabilidad de la muestra

En la figura 1 se muestra la evolución de la permeabilidad del testigo de mortero a lo largo del tiempo.

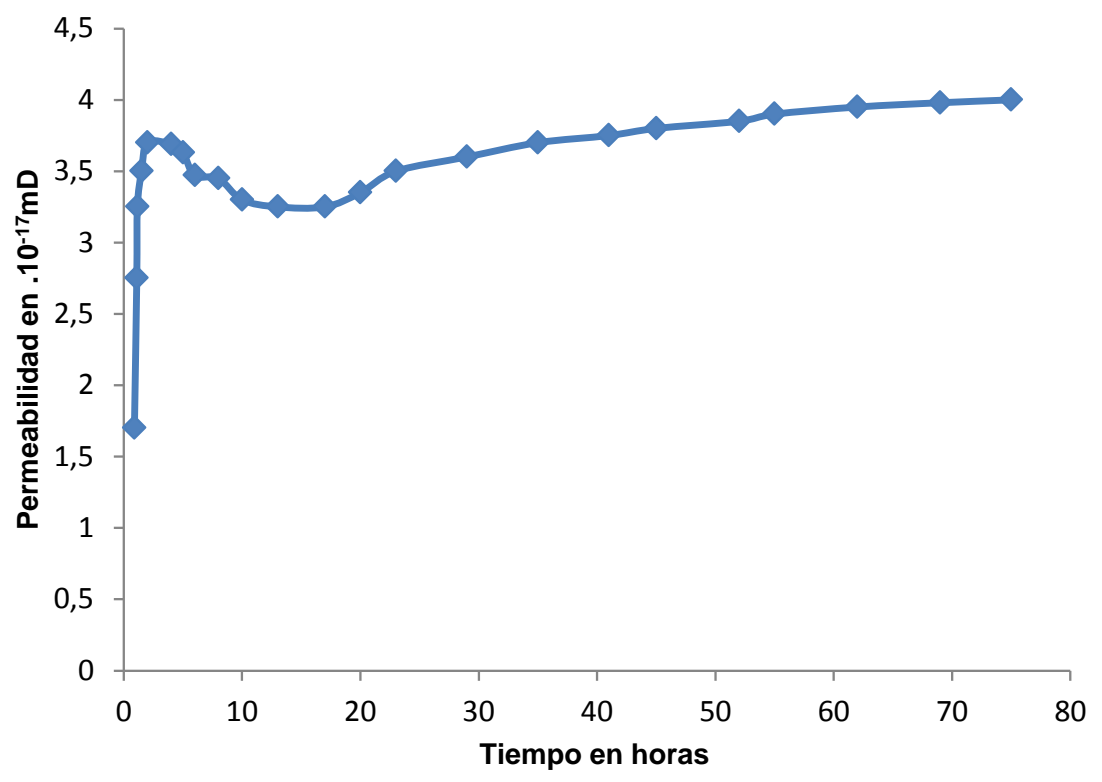

Fig. 1: Evolución de la permeabilidad del testigo

Como se puede observar, en las primeras horas de la realización del ensayo se produce un aumento rápido del caudal (figura 1). Esto se produce, por la saturación incompleta del testigo antes de iniciar el ensayo y por la absorción de agua por parte del ligante, proceso que disminuye paulatinamente.

Pasadas $2 \mathrm{~h} 30 \mathrm{~m}$, el caudal filtrado disminuye antes de estabilizarse y posteriormente crece ligeramente. Esta disminución del caudal filtrado se corresponde con la reorganización interna de las partículas en estado sólido libres dentro del testigo, bloqueándose algunas en los estrechamientos de los poros. Esta reorganización y el empuje de las más finas provoca un ligero aumento del caudal, y consiguientemente, de la permeabilidad del testigo. Lo que indica que empiezan a formarse los geles del tipo C-S-H responsables del fraguado del mortero. 


\section{Elementos arrastrados en la filtración}

Una vez realizado los ensayos de filtración y analizados las muestras de lixiviados obtenidas, se han identificado de los siguientes elementos, según aparece en la tabla 3. Para la mayor parte de los elementos, su concentración es poco importante en las primeras horas, tendiendo a cero hacia el final del ensayo. La sensibilidad de las mediciones de estos elementos es $\leq$ al $5 \%$.

Tabla 5: Elementos arrastrados en la filtración (Elaboración propia)

\begin{tabular}{|l|}
\hline Elementos \\
\hline $\mathrm{Si}, \mathrm{Na}, \mathrm{K}, \mathrm{Al}, \mathrm{Ca}, \mathrm{Li}, \mathrm{B}, \mathrm{Mg}, \mathrm{Sc}, \mathrm{Ti}, \mathrm{V}, \mathrm{Cr}, \mathrm{Mn}, \mathrm{Fe}, \mathrm{Co}, \mathrm{Ni}, \mathrm{Cu}$, \\
$\mathrm{Zn}, \mathrm{Ga}, \mathrm{Ge}, \mathrm{As}, \mathrm{Rb}, \mathrm{Sr}, \mathrm{Y}, \mathrm{Zr}, \mathrm{Nb}, \mathrm{Mo}, \mathrm{Pd}, \mathrm{Ag}, \mathrm{Cd}, \mathrm{Sn}, \mathrm{Sb}$, \\
$\mathrm{Cs}, \mathrm{Ba}, \mathrm{La}, \mathrm{Ce}, \mathrm{Pr}, \mathrm{Nd}, \mathrm{Sm}, \mathrm{Yb}, \mathrm{Lu}, \mathrm{Hf}, \mathrm{Ta}, \mathrm{Tl}, \mathrm{Pb}, \mathrm{Th}$ y U. \\
\hline
\end{tabular}

A continuación, se muestra cómo evoluciona la liberación de los elementos con mayores concentraciones presentes en el agua de filtración del mortero. Para la mayor parte de los elementos, su concentración es poco importante en las primeras horas, tendiendo a cero hacia el final del ensayo. La sensibilidad de las mediciones de estos elementos es $\leq$ al $5 \%$.

Los resultados obtenidos en estos ensayos se compararan con los límites máximos permitidos para los metales estudiados, para el caso de agua de uso doméstico, establecido en la legislación de Estados Unidos, la organización Mundial de la salud (OMS) y la Unión Europea (UE). Los datos se tomaron de la EPA, la OMS, y la directiva 98/83/EC de Europa, respectivamente. Para el caso del vanadio se ha empleado como referencia lo establecido en la legislación alemana con un valor máximo de 0,005 $\mathrm{mg} / \mathrm{l}$ en aguas superficiales previa potabilización. Los límites usados aparecen en la tabla 6.

Tabla 6: Límites máximos permisibles (mg/l). (Elaboración propia)

\begin{tabular}{|c|c|c|c|}
\hline Metales & EPA & OMS & UE \\
\hline $\mathrm{Zn}$ & 5.00 & 3.00 & 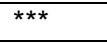 \\
\hline $\mathrm{Cr}$ & 0.10 & 0.05 & 0.05 \\
\hline $\mathrm{Pb}$ & 0.015 & 0.01 & 0.01 \\
\hline As & 0.05 & 0.01 & 0.01 \\
\hline $\mathrm{Cd}$ & 0.005 & 0.003 & 0.005 \\
\hline $\mathrm{Ni}$ & $\star \star \star *$ & 0.02 & 0.02 \\
\hline V & 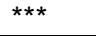 & $\star \star \star \star ~$ & 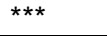 \\
\hline $\mathrm{Ba}$ & 2.00 & 0.30 & $\star \star \star *$ \\
\hline $\mathrm{Sr}$ & 4.00 & 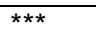 & $\star * *$ \\
\hline
\end{tabular}

\section{Concentración del sodio ( $\mathrm{Na})$}

De todos los elementos el liberado en mayor cantidad es el sodio $(\mathrm{Na})$, su concentración en las aguas filtradas se encuentra alrededor de los $5300 \mathrm{mg} / \mathrm{l}$ en las 5 primeras horas de ensayo. Dicha concentración a partir de este momento disminuye rápidamente hasta quedar alrededor de los $210 \mathrm{mg} / \mathrm{l}$ al finalizar el ensayo. En la Fig. 2 se muestra la evolución de la concentración del sodio a lo largo de tiempo del ensayo. El resultado obtenido para el sodio, se debe principalmente a que este elemento se encuentra en un porcentaje elevado en el vidrio utilizado $(\approx 11.3 \%)$, y además a que presenta alta movilidad. Por otra parte, el cemento CEM I, que constituye el $20 \%$ del ligante, no contiene más que un $0.15 \%$ de sodio, aportando muy poco a la concentración.

\section{Concentración de silicio y potasio}

En la figura 3 se observa que la liberación del potasio en las aguas filtradas del mortero alcanza una concentración inicial mayor de unos $350 \mathrm{mg} / \mathrm{l}$ y que disminuye rápidamente hasta quedar alrededor de 30 $\mathrm{mg} / \mathrm{l}$ al finalizar el ensayo. Este elemento proviene tanto del vidrio como del cemento, cuyo contenido en potasio es respectivamente el $0.6 \%$ y $0.7 \%$.

En cuanto a la disolución del silicio, su concentración disminuye de forma más lenta que en los casos ya expuestos del sodio y del potasio, por lo que su cinética es algo diferente. Por otra parte la diferencia entre las concentraciones del principio y el final del ensayo es significativamente menor en el caso del silicio, ya que varía con un coeficiente de 3, mientras que en el caso del sodio esta cercano al 25 y al 11 en el caso del potasio. El silicio, aunque es constitutivo del vidrio en un $70 \%$, es un elemento que se disuelve con mayor dificultad que los elementos alcalinos. También el cemento aporta un $19 \%$ de silicio a la disolución. 


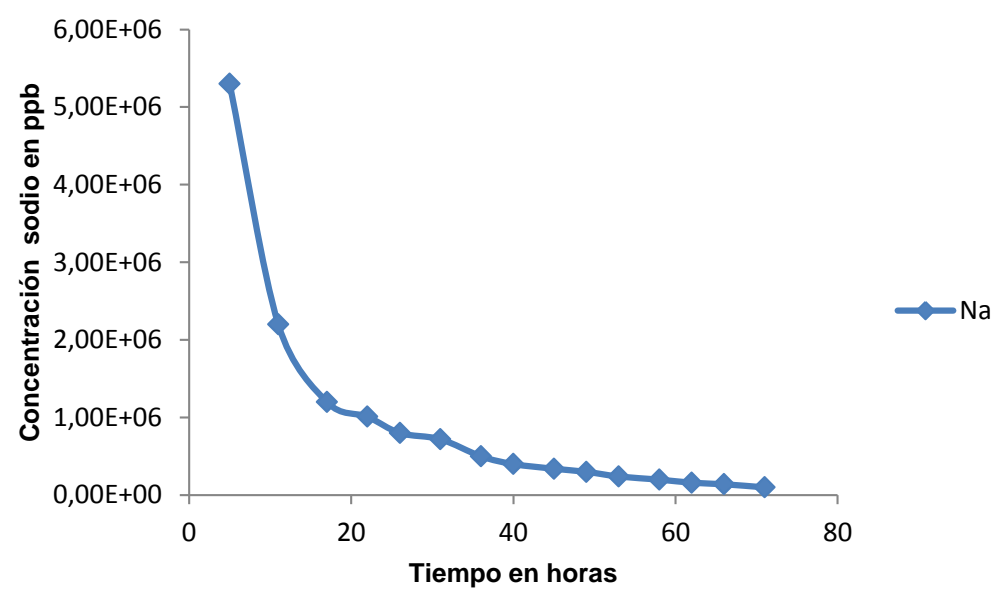

Fig. 2: Variación de la concentración de sodio

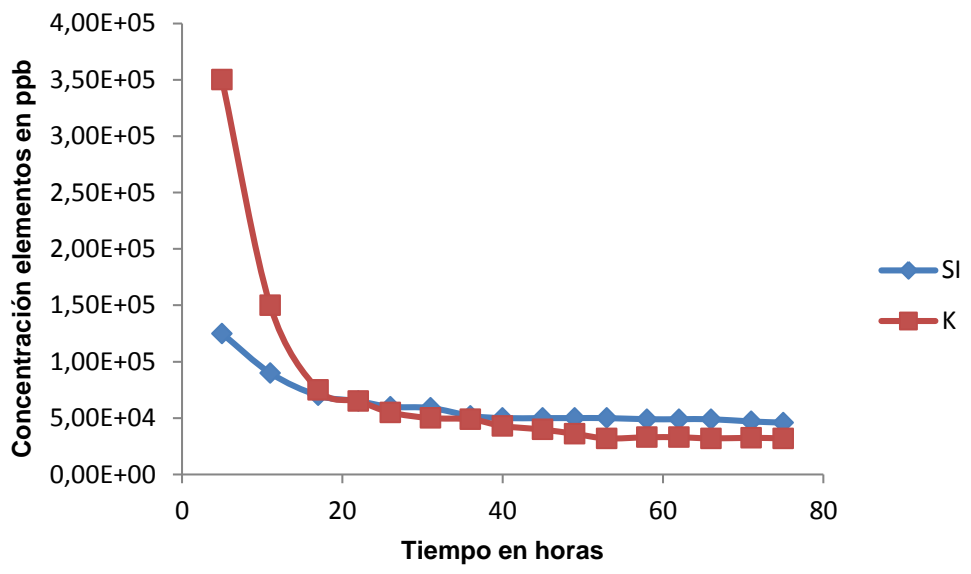

Fig. 3: Variación de la concentración de silicio y potasio

Variación de la concentración de aluminio

El aluminio es otro componente del vidrio sodo-cálcico en un porcentaje de un 2.2\%, por lo que también aparece en las aguas filtradas. La concentración de este elemento disminuye rápidamente según se ve en la Fig. 4 desde los $18 \mathrm{mg} / \mathrm{l}$ hasta $1.6 \mathrm{mg} / \mathrm{l}$. El cemento constituido por un $5.5 \%$ de aluminio, también aporta cierta cantidad.

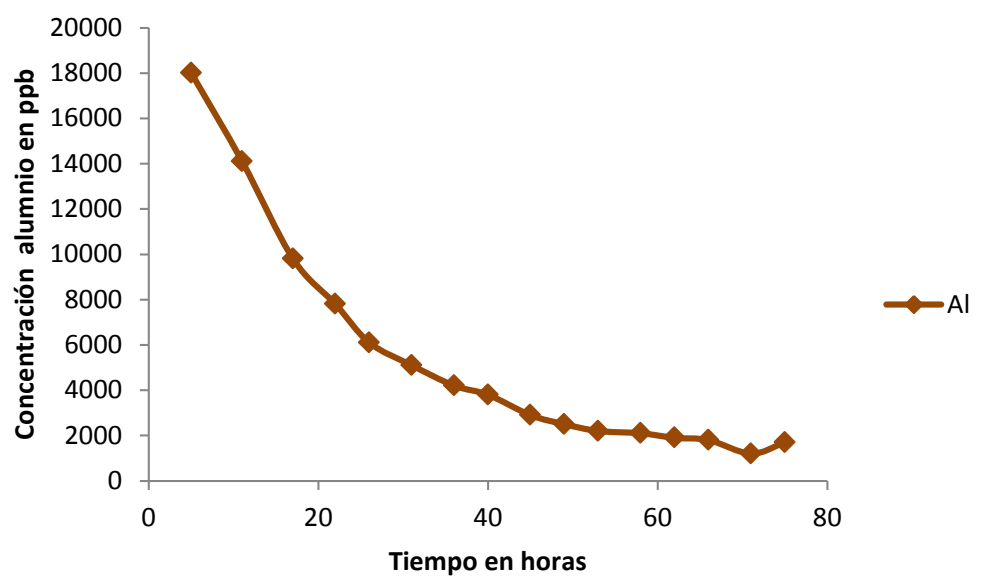

Fig. 4: Variación de la concentración de aluminio.

Variación de la concentración del calcio, cromo y boro

Las concentraciones de calcio según queda reflejado en la figura 5, varían de forma diferente a lo largo del tiempo. En el inicio del ensayo se produce una disminución de la concentración de calcio pasando de 3.2 $\mathrm{mg} / \mathrm{l}$ a $1.3 \mathrm{mg} / \mathrm{l}$. Posteriormente la concentración se mantiene constante hasta que transcurren 30 horas. Después de este tiempo, la concentración sufre un aumento hasta alcanzar un valor final de $3.8 \mathrm{mg} / \mathrm{l}$. 


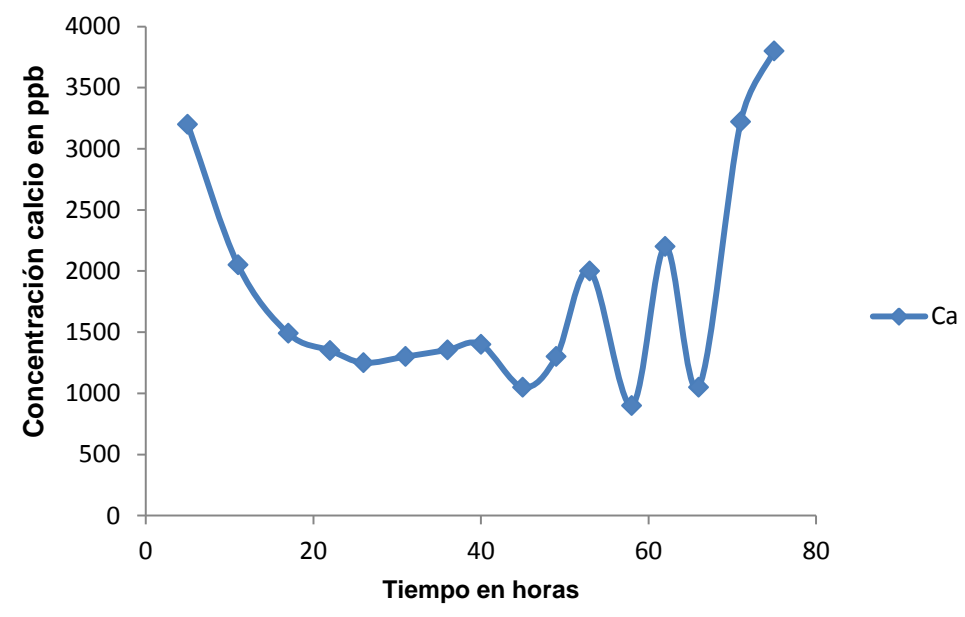

Fig. 5: Variación de la concentración de calcio

Según Marco et al. (2012), la disolución del vidrio no liberaba calcio, por lo que se puede concluir que el recogido en la filtración proviene en gran medida del cemento, ya que el cemento está constituido por un $65 \%$ de $\mathrm{Ca}$. El Ca liberado por disolución del vidrio reacciona inmediatamente con los otros elementos constitutivos del vidrio para formar los geles del tipo $\mathrm{C}-\mathrm{S}-\mathrm{H}$, responsables del fraguado del mortero. Lo que explica la ausencia de calcio en las soluciones. Ello implicaría igualmente que la concentración de calcio en las soluciones sería el parámetro limitador de la formación de los geles del tipo C-S-H.

El vidrio molido multiplica las superficies reactivas, lo que provoca un aumento de la velocidad de reacción. Teniendo en cuenta su composición química, su estructura amorfa y su pequeño tamaño, las partículas de vidrio reaccionan muy rápidamente con los hidrófilos en solución liberando sílice, sodio y calcio que se organizarán para dar lugar a la aparición de los geles arriba mencionados. Después de todos los datos obtenidos, el fraguado provocado por el vidrio molido es debido a la formación de los geles tipo C-S-H. Dichos geles son los responsables de que el fraguado se prolongue en el tiempo en comparación con los morteros cemento convencionales y confiere a los suelos estabilizados con este tipo de morteros la característica de la autoreparabilidad cuando se presentan deformaciones (Marco et al, 2012). En la Figura 6 aparecen reflejadas las concentraciones del cromo y del boro.

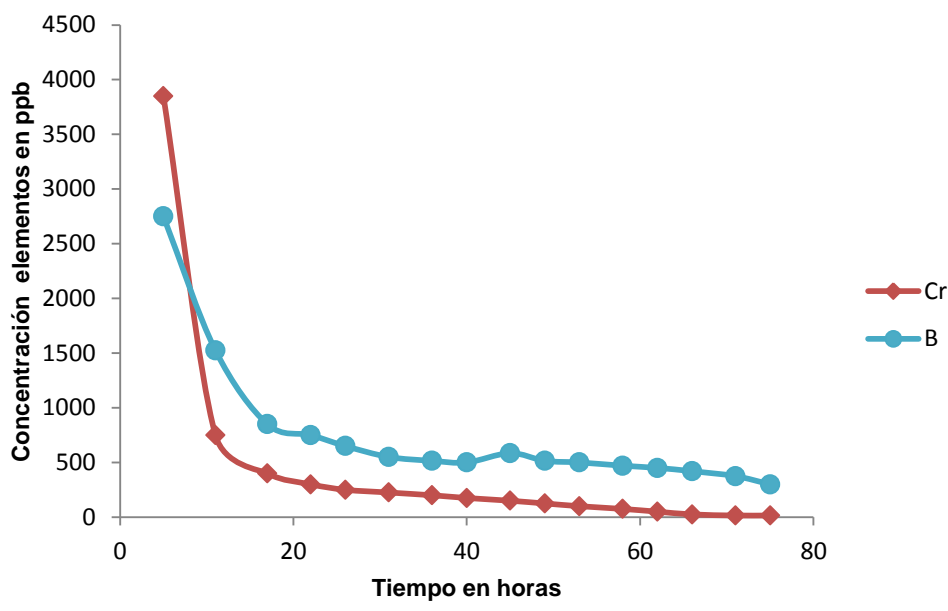

Fig. 6: Variación de la concentración de cromo y boro

La concentración de boro es apreciable al principio del ensayo, disminuyendo rápidamente con el tiempo pasando de $2.7 \% \mathrm{mg} / \mathrm{l}$ a $0.3 \% \mathrm{mg} / \mathrm{l}$. Este elemento proviene del vidrio.Otro de los elementos que se detecta en las primeras horas, es el cromo, con una concentración de $3.7 \mathrm{mg} / \mathrm{l}$. Esta concentración disminuye rápidamente hasta quedar en una concentración de $0.08 \mathrm{mg} / \mathrm{l}$ al finalizar el ensayo. La presencia del cromo es debido a que en el lote de vidrio utilizado aparece este elemento en una cantidad de $843 \mathrm{mg} / \mathrm{kg}$. El cromo en el vidrio es utilizado para conferir a éste propiedades características. Es conveniente recordar, como ya se expuso en el apartado de Materiales y Métodos, que los ensayos de filtración se han realizado con una presión de inyección de agua sobre el testigo de 10 bares, lo que provoca un lavado fuerte de los elementos en las aguas filtradas. 


\section{Variación de concentración de hierro, cobre, vanadio, níquel y molibdeno}

En la figura 7, se observa una concentración de los elementos hierro, cobre, vanadio, níquel y molibdeno más alta en las primeras horas del ensayo, que tienden a disminuir hasta quedar estabilizadas transcurridas $20 \mathrm{~h}$, resultando prácticamente nulas al finalizar el ensayo. Las variaciones de concentración que poseen estos elementos son comparables a las del sodio y el potasio, pero con valores más pequeños.

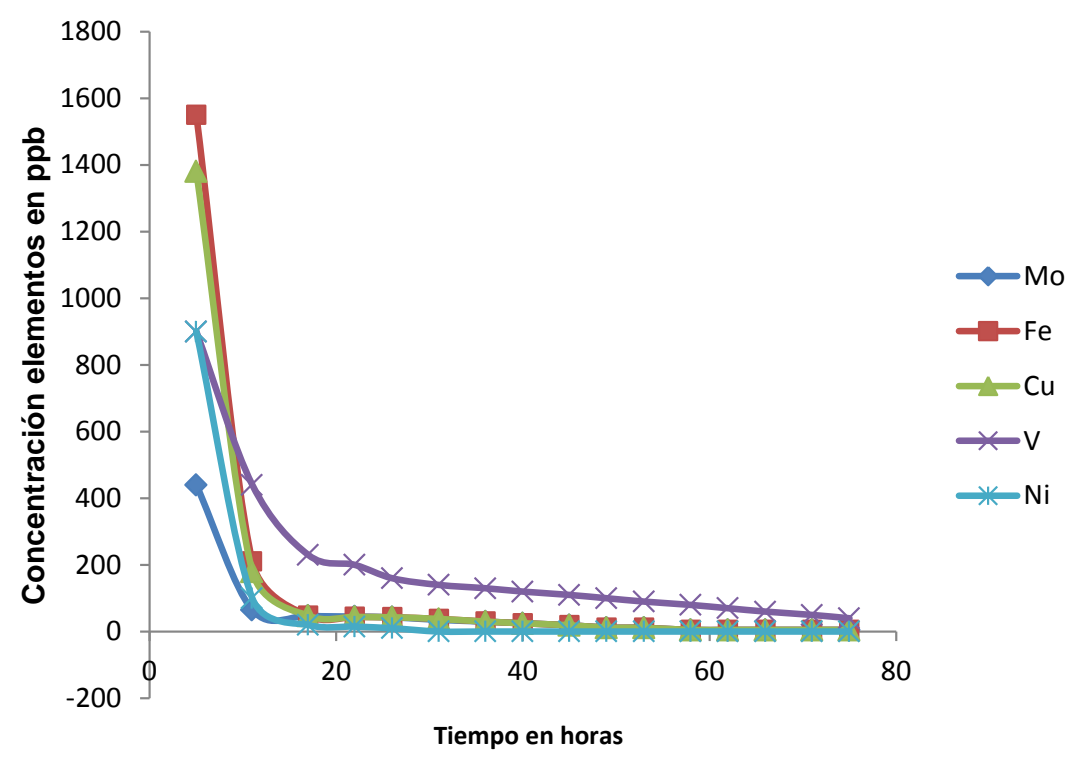

Fig. 7: Variación de la concentración de hierro, cobre, vanadio, níquel y molibdeno

Los demás elementos arrastrados en la filtración (tabla 3), tienen cinéticas de disolución comparables a las del sodio y el potasio, pero con inferiores concentraciones $(<0.2 \mathrm{mg} / \mathrm{l})$ al principio del ensayo bajando muy rápidamente hasta quedar estabilizadas en cantidades imperceptibles al finalizar el mismo.

\section{CONCLUSIONES}

De los resultados mostrados, de su análisis y su discusión, se pueden obtener las siguientes conclusiones, sobre el impacto ambiental producido por la utilización de morteros fabricados con polvo de vidrio como ligante:

i) La liberación por lavado de elementos ha demostrado que los elementos alcalinos (sodio y potasio), sobre todo el sodio proveniente principalmente del vidrio, es liberado en cantidad importante, pero este no resulta un peligro para el medio ambiente, ya que no se libera en cantidad suficiente para producir modificaciones en la conductividad eléctrica de suelo.

ii) La presencia de $\mathrm{Ca}$ en el agua de filtrado, no constituye un peligro medioambiental, ya que es un elemento no tóxico y en las concentraciones medidas no provocaría modificación del pH del suelo.

iii) Igualmente es necesario destacar la presencia considerable de cromo y de boro en las aguas de filtrado, elementos potencialmente contaminantes, durante las primeras horas del ensayo. Dichos elementos tienden a desaparecer a la finalización del mismo, ya qie quedan incorporados en la matriz cementicia por lo que no provocan contaminación ambiental.

iv) El resto de elementos detectados no son más que huellas contenidas en las aguas filtradas que por su escasez no pueden constituir un peligro para el medio natural según la legislación empleada.

v) La utilización de desechos de polvo de vidrio abre perspectivas interesantes en cuanto a la sostenibilidad en la ingeniería civil, ya que estos morteros fabricados con ligante de polvo de vidrio, pueden ser utilizados en el sector de firmes de carreteras, morteros o en tratamiento de suelos finos.

vi) Se valorizan los desechos de polvo de vidrio que de no ser utilizados se depositarían en vertederos, con el consiguiente beneficio económico-ambiental.

vii) El uso de morteros fabricados con ligante de polvo de vidrio cumple con los criterios de sostenibilidad que la sociedad actual demanda. Ya que por un lado disminuye la cantidad de cemento a emplear, con lo que se contribuye a la disminución de la emisión de gases de efecto invernadero, y por otro disminuye la superficie destinada a vertederos de inertes. 


\section{REFERENCIAS}

Ahmad S., Aimin X., Value-added utilisation of waste glass in concrete, doi:10.1016/S0008-8846(03)002515, Cement and Concrete Research, 34, 81-89 (2004)

Bashart T., Ghassan N., "Properties of concrete contains mixed colour waste recycled glass as sand and cement replacement". doi:10.1016/j.conbuildmat.2007.01.019, Construction and Building Materials, 22(5), 713-722 (2008)

Burgueño Muñoz, A., La evaluación de la sostenibilidad en obra civil, Actas de $5^{\circ}$ Congreso Nacional de Ingeniería Civil: Desarrollo y Sostenibilidad en el Marco de la Ingeniería, 1-14, Sevilla, España 26 a 28 de Noviembre (2007)

Cánovas, M. Hormigón. 10ª Edición. 1-669. Ibergarceta S.L., Madrid, España, (2013)

Directrices de la OMS para la calidad del agua potable. (en línea: http:// www.aguaesalud.com/directricesOMSaguapotable.html, acceso: 18 de Diciembre de 2015), Organización Mundial de la Salud (2008)

Gonzalo A. Valdés y Jorge G. Rapimán. Propiedades Físicas y Mecánicas de Bloques de Hormigón Compuestos con Áridos Reciclados, doi: 10.4067/S0718-07642007000300010, Información Tecnológica (en línea), 18 (3), 81-88 (2007)

Marco, L. J., García, E., Más, M.I. et al, Estudio de la resistencia a compresión de morteros fabricados con conglomerante compuesto de polvo de vidrio, doi: 10.3989/ic.11.100, Informes de la Construcción (en línea), 64, 529-536 (2012)

Martínez, C. et al., Uso de test de lixiviación para determinar la migración de contaminantes en morteros de sustitución con residuos de catalizador de craqueo catalítico (fcc), doi: 10.15446/dyna, Dyna (en línea), 181, 163-170, (2013)

Rodríguez, F., Fernández. G. Ingeniería sostenible: nuevos objetivos en los proyectos de construcción, Ingeniería de Construcción, ISSN: 07185073, (en línea; http://www.ricuc.cl/index.php/ric, acceso Enero 17 2015), 25,147-160 (2010)

Directiva 98/83/EC, Estándares Europeos de la calidad del agua potable. Sobre la calidad del agua destinada a consumo humano, (en línea: http://www.lenntech.es/aplicaciones/potable/normas/estandareseuropeos-calidad-agua-potable.htm\#ixzz13C1IJX00. Acceso: 18 de Diciembre de 2015)

UNE-EN 12390-8, Ensayos de hormigón endurecido. Parte 8: Profundidad de penetración de agua bajo presión, 5-8, Madrid, España (2009)

UNE-EN 196-1, Métodos de ensayo de cementos. Parte 1: Determinación de resistencias mecánicas, 20-23, Madrid, España (2005)

US EPA 815-F-00-007, Estándares del Reglamento Nacional Primario de Agua Potable., DC:U.S. Environmental Protection Agency, 1-8, Washington, USA (2000)

Wirtschafts, U., Verlag-ges.,Gas Wasser, U., Daten und Informationen zu Wasserinhaltsstoffen, DVGWSchriftenreihe WASSER, 1985-1988, Eschborn, Deutschland (2002) 
